\title{
LA MEMORIA, LA TRACCIA E LA FUNZIONE \\ DEL NARRATORE \\ in “Ritorno sul Don” Di Mario Rigoni Stern
}

\section{Gianluca Cinelli}

\begin{abstract}
Riassunto: L'esperienza della guerra combattuta in Russia nel 1942-1943 è centrale nell'opera di Mario Rigoni Stern. In questo articolo l'attenzione si concentra sul racconto "Ritorno sul Don" (1973) in cui lo scrittore, narrando il viaggio compiuto all'inizio degli anni Settanta sui luoghi dove aveva combattuto, scopre l'importanza delle tracce in cui il passato si incide nel paesaggio e nella memoria. In riferimento a un'osservazione di Paul Ricoeur, e soprattutto alla filosofia nietzschiana dell'eterno ritorno, sarà interpretato il modo in cui la traccia fonda qui la testimonianza di un passato che il narratore vivifica come verità celata sotto l'apparenza del presente, mostrando la morte come il confine metaforico oltre il quale l'esperienza diventa racconto, memoria e testimonianza.
\end{abstract}

L'esperienza della guerra è centrale nell'opera di Mario Rigoni Stern, insieme con la diffusa ispirazione autobiografica intuita da Vittorini, che scrisse sul risvolto di copertina della prima edizione de Il sergente nella neve nella collana "I gettoni" (1953): "Mario Rigoni non è scrittore di vocazione ... alpinista, cacciatore, impiegato statale, forse non sarebbe capace di scrivere di cose che non gli fossero accadute". Infatti Mario Rigoni Stern è per vocazione piuttosto un narratore di storie tratte dall'esperienza vissuta e dalla tradizione trasmessa di bocca in bocca, storie conosciute nell'arco di una vita, dai paesani, dai malgari e dai boscaioli nella sua terra d'Asiago, o dagli alpini incontrati negli anni della guerra, una tradizione orale che s'estingue e la cui sopravvivenza Rigoni Stern rimette alle forme della scrittura.

La guerra è il tema centrale de Il sergente nella neve, che Eraldo Affinati definisce "il nucleo originario di tutte le successive opere dello scrittore" ("Mario Rigoni Stern", xii) e si ripresenta infatti nel tempo in quasi tutti i suoi libri, ora come allegoria del passaggio dalla giovinezza alla maturità soprattutto nel recente Lultima partita a carte (2002) —, ora come "metafora ossessiva" della "perdita della natura" 1 La guerra de Il sergente

${ }^{1}$ Motta, Mario Rigoni Stern, 15. L'espressione "metafora ossessiva" ricorda quella coniata da Charles Mauron, Dalle metafore ossessive al mito personale, in un con- 
nella neve è una "condizione abnorme di uomini contro uomini, di forti contro deboli” (Motta, Rigoni Stern,15), esperienza della separazione dell'umano dalla natura, intesa in senso ampio come physis. la testimonianza di una simile esperienza si configura metaforicamente come un attraversamento del confine fra la vita e la morte, che il narratore compie tributando un incessante "dovere di memoria" a coloro che non sono tornati. Come vedremo, Rigoni Stern assume proprio la morte come limite estremo dell'esperienza e condizione perché questa diventi racconto, sicché la sua testimonianza del passato è un metaforico dialogo con il tempo in cui riposano i morti, dialogo che può essere inteso con le parole di Motta, come lo "scardinamento di un ordine naturale" (Mario Rigoni Stern, 15) e che nel racconto "Tre patate lesse" (1973) si mostra in tutto il suo orfismo: "a me stesso io ero morto e cercavo disperatamente gli amici morti. Come avrei potuto, altrimenti, ripassare le Alpi?" (Rigoni Stern, Il sergente nella neve, 214)2.

Se Il sergente nella neve è il nucleo originario della memorialistica rigoniana, è ancora Affinati a riconoscere con puntualità che Ritorno sul Don (1973) ne è il "baricentro strutturale" (Affinati, "Mario Rigoni Stern", xxiii) che conduce l'ispirazione autobiografica in una struttura a mosaico ${ }^{3}$, dove la

testo teorico affatto diverso da quello qui dispiegato: la metafora ossessiva è, secondo Mauron, l'insorgenza ripetitiva all'interno di un'opera o di un gruppo di opere di un autore di un contenuto psichico riconducibile a determinati temi. Mediante la sovrapposizione dei testi come "fotografie di Galton" (33), queste ripetizioni segnano una traccia che il critico segue fino a individuare delle "reti associative" e da queste a risalire fino al "mito personale", che è "una situazione drammatica interna, personale, incessantemente modificata per reazione agli avvenimenti interni o esterni, ma persistente e riconoscibile" (255). Forse Motta ha presente la teoria mauroniana quando scrive, comunque si tratta di un taglio metodico che non sarà seguito dal presente articolo.

${ }^{2}$ Secondo Michele Buzzi (1985), le tre patate lesse del racconto omonimo sono simbolo della baita lontana (espresso dal calore) e della terra che accomuna e affratella gli uomini. Tanto la baita quanto la terra sono a loro volta allegorici del movimento di rigenerazione dalla morte, che costituisce il nucleo della poetica rigoniana.

${ }^{3}$ È Affinati a sostenerlo, ma senza chiarire se questa struttura a mosaico riguardi Ritorno sul Don in quanto raccolta di racconti, oppure l'intera opera rigoniana, che distende l'autobiografia nel tempo e attraverso più testi montati in un'unica, ininterrotta scrittura del sé. Né, d'altra parte, Affinati specifica se si riferisce alla raccolta intera o al racconto che dà il titolo all'opera. In questo articolo si indica con il corsivo il titolo della raccolta, e l'attenzione è dedicata in partico- 
guerra, la natura e la poetica dell'eterno ritorno, ovvero della compenetrazione fra il tempo autobiografico e individuale in cui si dispiega il senso dell'esperienza vissuta, e il tempo immobile della physis che continuamente torna a ripetersi diversa eppure uguale, si costituiscono in un unico afflato narrativo. La raccolta di storie del 1973 , nata dal ritorno dello scrittore nei luoghi della sua Russia e della sua guerra, dispiega una poetica alla cui base sta la dialettica fra destino individuale e autobiografico e mito dell'eterno ritorno della vita a se stessa: la poetica della traccia.

La traccia è, dal punto di vista della coscienza storica, il segno dell'esistenza di un passato, è la manifestazione del passaggio di eventi, di esistenze, di vita, e la sua natura è eterogenea, perché ogni documento è prima di ogni altra cosa una traccia che un'epoca lascia di sé più o meno consapevolmente; ma la traccia è il segno di un passato che appunto torna continuamente nelle immagini della memoria. Per mezzo delle tracce, quindi, il passato si rende sempre attuale pur restando qualcosa di diverso e distante, e premendo sul presente appare agli occhi della coscienza stori$\mathrm{ca}$, come tradizione. La testimonianza di Rigoni Stern si mostra quindi come ascolto di una tradizione che riposa nelle tracce del passato, e nel frattempo questo stesso ascolto, che è un ricevere e un raccogliere, appare come la condizione del risorgere. Tale movimento, già oggetto in antichità delle riflessioni di Platone e di Aristotele, è descritto dalle moderne teorie dell'ermeneutica filosofica, soprattutto da Gadamer in Verità e metodo (1960), che descrive il rapporto fra presente e passato come dialettica dell'ascolto. Vedremo quindi come la poetica elaborata da Rigoni Stern per la prima volta in modo sistematico nel racconto "Ritorno sul Don", abbia carattere simbolico e allegorico in relazione rispettivamente alla traccia e al discorso della memoria. In questo racconto, infatti, sono messe fra loro in relazione dialettica l'esperienza autobiografica individuale, che presenta la struttura lineare e discontinua della coscienza storica, la quale conosce il passato mediante le sue tracce, e l'esperienza filosofica e mistica dell'eterno ritorno della physis a se stessa, mediante i cicli della morte e della rigenerazione. Il simbolismo della traccia è così trasceso in esperienza filosofica, quella che Benjamin chiama "l'esperienza del conio divino" ${ }^{4}$, cioè la gnose-

lare al racconto "Ritorno sul Don" perché, nell'economia dell'opera, come nota bene Buzzi pur non apprezzandola molto, tale brano si discosta dai primi, che sono ambientati nel passato della guerra, e da "La scure", che con l'allegoria della malattia come manifestazione della colpevolezza del sopravvissuto, funge da cerniera fra i racconti di guerra e la cronaca del pellegrinaggio di omaggio ed espiazione che è "Ritorno sul Don" (Invito alla lettura, 69).

${ }^{4}$ Benjamin, Angelus novus, 166. 
ologica penetrazione del senso celato nell'apparenza delle forme.

Ma in che modo intenderemo l'idea di eterno ritorno espressa nel racconto "Ritorno sul Don"? Consideriamo due accezioni diverse, l'una datane da Nietzsche ne La gaia scienza (1882), Cosi parlò Zarathustra (1885), Il Crepuscolo degli idoli (1888) e La volontà di potenza (1906), come l'impulso vitalistico, definito anche come "volontà di potenza", di accrescersi al di là della finitezza, che è proprio dei viventi. Un impulso che però, nella teoria nietzschiana, non deriva da una volontarietà o intenzionalità della coscienza o dell'lo (contro la tradizione cartesiana e hegeliana), e che dunque non osserva il movimento finalistico e lineare di sviluppo da un punto di partenza a uno di arrivo, ovvero non ha un carattere metafisico. Secondo Nietzsche il ritorno è eterno in quanto i fenomeni, avvenendo in un sistema finito (il cosmo) $\mathrm{ma}$ in un tempo non limitato, sono soggetti ad accadere infinite volte. Si tratta quindi di un "principio dionisiaco", cioè di

un impulso all'unità, un andare al di là della persona, del quotidiano, della società, della realtà... un'estatica affermazione del carattere totale della vita, un carattere uguale in tutti i mutamenti, sempre ugualmente potente e felice; la grande simpatia panteistica del gioire e del soffrire, che approva e santifica persino le qualità più terribili ed enigmatiche della vita per una eterna volontà di creazione, di fecondità, di eternità, per il senso dell'unità necessaria del creare e del distruggere... (Nietzsche, La volontà di potenza, 551)

Eterno ritorno non significa per Nietzsche immobilizzazione nell'eternità cristallina della forma assoluta, ma trascendimento della forma contingente, mediante la sua distruzione e morte, in vista di un senso più alto e più completo, ma mai definitivo, di totalità e unità della vita.

L'altra accezione è quella antropologica di Mircea Eliade, come rappresentazione del tempo mitico nell'immaginario delle culture arcaiche. Nella teoria di Mircea Eliade, l'uomo arcaico è solidale col cosmo e con i suoi cicli, a differenza di quello moderno che invece rappresenta la realtà in termini di storia. Anche il cosmo degli arcaici ha una storia, ma essa è il mito, è storia sacra ripetibile nel mito, cosicché ogni azione è già stata fatta, ogni gesto umano è ripetizione. Per il pensiero mitico il "centro" è il luogo della realtà assoluta e anche il "sé" partecipa di questo centro, e poiché il mito e la ripetizione aboliscono il tempo, la ripetizione non è riproduzione in immagine dell'evento primordiale, ma coincidenza in esso5 ${ }^{5}$. A nostro avviso la poetica rigoniana intreccia alcuni motivi di entrambe le accezioni, e

${ }^{5}$ Eliade, Il mito dell'eterno ritorno, 56. Sebbene Mircea Eliade non manchi di indi- 
vedremo come ciò si rivela nella forma della narrazione mediante la sovrapposizione di fenomeni e rappresentazioni appartenenti al passato e al presente, e tenuti insieme nel riconoscimento della traccia come punto in cui appunto passato e presente sono uniti in una coestensione temporale. Tuttavia la visione di tale temporalità contraddittoria (eternità della physis e finitezza dell'esistenza) si discosta dall'idea di eterno ritorno di Mircea Eliade, nella misura in cui la ripetizione dei cicli cosmici (espressa nel racconto come rinascita della vita in Russia dopo la morte causata dalla guerra, oppure nel simbolo della neve) è esperita attraverso il filtro della coscienza storica, cioè all'interno di una temporalità lineare in cui il passato e il presente si differenziano. Quindi l'eterno ritorno così come appare nella poetica rigoniana rievoca soprattutto la metafora nietzschiana del principio "mistico" della "unità necessaria del creare e del distruggere", della "estatica affermazione del carattere totale della vita". È nello iato fra finitezza ed eternità, fra distruzione e rigenerazione, che l'individuo riceve storia e identità, e questo iato impedisce al "sé" dell'esperienza vissuta e rappresentata nella memoria di coincidere con il mito. La memoria diventa così coscienza storica, ovvero coscienza della finitezza umana e dell'impossibilità di coincidere con il "centro". La poetica rigoniana condivide maggiormente l'inquietudine della prospettiva nietzschiana, in cui l'uomo scopre di trascendere se stesso costantemente senza che ciò dipenda dalla sua volontà soggettiva. Pertanto riecheggia, come vedremo meglio con riferimenti diretti al testo, la nostalgia per la perdita della "pienezza" di una vita infranta dallo iato dell'esperienza vissuta della guerra, metafora per eccellenza della "perdita della natura", come sostiene Motta. Alla luce di ciò assume nuovo senso l'osservazione di Buzzi, secondo cui Ritorno sul Don (ma in particolare il racconto omonimo) non è un libro sereno, ma "un libro mesto e dolente, impregnato di una irrequieta e amara nostalgia" (Buzzi, Invito alla lettura, 69).

care che la mentalità arcaica non disconosce un tempo storico, in cui si susseguono eventi come guerre, carestie e peccati individuali, ma tenta di evaderlo come tempo della sofferenza, del caos e della morte. Ma anche qui Rigoni Stern si distanzia: nelle sue opere di memorialistica, sia quelle maggiori Il sergente nella neve e Quota Albania (1971), sia i racconti de Il bosco degli urogalli (1962) e di Ritorno sul Don, il tempo storico non è soltanto dolore, non c'è solo la guerra a scandire i giorni, ma anche il lavoro, la caccia, la vita dei boschi e dei monti. E anche quando rappresenta la guerra, Rigoni Stern concede spazio alle scene di tregua e di serenità, spesso mostrando la dimensione quotidiana della vita e del lavoro dei soldati al fronte. Si pensi, a titolo non esclusivo di esempio, alla prima parte de Il sergente nella neve, "Il caposaldo". 
Torniamo dunque a fare alcune osservazioni sulla funzione ermeneutica della traccia, che Ricoeur argomenta a partire dalle riflessioni platoniche sull'enigma dell'eikon, dell'immagine che rende presente l'assente (Ricoeur, Ricordare, 9-19)6 Infatti nella traccia non si materializza il passato, né in essa si manifesta la sua causa, la quale però è evocata come necessità della sua presenza; quindi l'enigma, argomenta Ricoeur, è duplice, in quanto enigma della presenza dell'assente (evocazione), e del suo contenuto di verità (la somiglianza dell'impronta all'originale). Secondo Paul Ricoeur, dietro la traccia è sempre celata una volontà del tracciare, una presenza alternativa alla traccia stessa e a chi la contempla (Ricoeur, Ricordare, 12) e tale anteriorità è il segno che qualcosa è accaduto, è una testimonianza di vita, di esperienza, un deposito di potenziale conoscenza del passato. Questa essenza storico-materiale della traccia come segno in cui il significato del passato si sedimenta, è ben presente nell'opera di Rigoni Stern. Armi, indumenti, oggetti, scheletri, bombe inesplose nelle trincee abbandonate della Grande Guerra, affollano la memoria di Rigoni Stern, il quale trova nella loro materialità un duplice rimando alla storia del conflitto, di coloro che vi combatterono provenendo da terre lontane e della gente che ebbe sconvolta la vita dall'arrivo del fronte sui propri territori e nelle proprie case ${ }^{7}$; allo stesso tempo i cimeli richiamano il ricordo dell'infanzia trascorsa fra i monti, alla scoperta della tradizione e della natura, rispetto alle quali si articola il tempo autobiografico, tempo dell'infranto e del finito. Ora, però, non rischia di essere contraddittorio descrivere la poetica rigoniana, contemporaneamente come reminiscenza ed eterno ritorno? L'esperienza della traccia è sicuramente all'origine di reminiscenza, ma di che cosa? Vedremo che in "Ritorno sul Don" l'oggetto del ricordo sono immagini, rappresentazioni, forme. Ciò che la traccia richiama non è un archetipo, niente di superiore o migliore della traccia stessa, del presente, dell'esperienza presente. Il passato che affio-

${ }^{6}$ Ricoeur fa riferimento esplicito al Sofista e al Teeteto di Platone, ma l'enigma della traccia (dell'impronta in Platone) richiama anche necessariamente il contesto filosofico della preesistenza dell'anima immortale e della reminiscenza del mondo delle idee, quindi di altre opere quali Fedone e Fedro. Noi non seguiremo Ricoeur in questa direzione, ma tenteremo di mostrare che l'enigma della traccia è posto nel racconto di Rigoni Stern in termini non di una metafisica idealistica, bensì di una filosofia del divenire, in cui la ripetizione dell'uguale nell'apparenza dei fenomeni si manifesta come rappresentazione, cioè come finitezza formale infinitamente generatrice di senso, come argomentato nella sezione "La volontà di potenza come rappresentazione", de La volontà di potenza.

7 Come nei racconti di Rigoni Stern, "Cartoline" (1999) e "La bottiglia ritrovata" (2005). 
ra nei segni della storia, del paesaggio, della cultura non cessa mai di essere esperienza umana, e perciò qualcosa di finito, parziale, interrotto. Tuttavia ognuna di queste forme può tornare infinite volte nel tempo, nelle tracce, nei ricordi, nei racconti. Perciò non è contraddittorio riconoscere nella poetica rigoniana della traccia il movimento ciclico della memoria come reminiscenza (dal passato al presente) e dell'eterno ritorno (dal presente al passato). Il complesso delle rappresentazioni coinvolte in questo movimento è la tradizione, orizzonte originario della coscienza storica. Infatti, il passato rievocato dalle tracce non appartiene all'individuo che ricorda, come un contenuto della sua coscienza: al contrario è l'esperienza della traccia ad appartenere ad una memoria non soggettiva, della tradizione appunto, di cui il contenuto individuale è soltanto un momento e un punto di vista limitato, una specie di interruzione. Perciò quella tradizione, che sprofonda nel passato fino a confondersi con la vita dei luoghi in cui si tramanda, appare come l'eterno ritorno di una pienezza e di una continuità in cui la memoria individuale del narratore si cala con l'anelito a dissolversi in esse.

Tuttavia il senso della traccia non si esaurisce nell'essere il punto di contatto ermeneutico fra la tradizione e il presente, e nelle tracce della Grande Guerra Rigoni non trova soltanto i segni della storia e delle sue conseguenze rispetto alle tradizioni millenarie della comunità di Asiago. Esse non sono soltanto i cimeli di una archeologia autobiografica in quanto testimonianze della società, della vita e della cultura che ha preceduto, preparato e accolto l'esistenza dello scrittore: molto più che le impronte lasciate dagli avi di Rigoni Stern, esse sono anche i segni materiali in cui si imprime l'essere nel suo divenire, "carattere uguale in tutti i mutamenti" appunto in accordo con la teoria nietzschiana, sedimentazioni delle infinite possibilità, sempre diverse eppure uguali, con cui l'essere si manifesta nel tempo della finitezza umana. La vita dei boschi, le trasformazioni del paesaggio, l'estensione di pascoli e ghiacciai, l'apertura di nuove strade sui fianchi dei monti, anche queste sono tracce in cui la storia può riecheggiare, ma che indicano il divenire di una inesauribile continuità della natura, rispetto alla quale la storia non è che un accadere di possibilità.

La traccia è ciò mediante cui il narratore mostra di appartenere al passato e tanto più diventa importante quanto maggiore è la sua capacità di risvegliare ricordi, connettere racconti slegati, indicare nuove vie da percorrere in cerca del senso del divenire. Lo stile narrativo di Rigoni Stern si ispira ai modelli dell'oralità, nei quali la relazione fra racconto e memoria è strettissima. Su questo punto lo stile rigoniano si fa più prossimo al mito, con il quale condivide un aspetto fondamentale: l'esperienza vissuta e l'esperienza collettiva, tramandate al racconto rispettivamente per mezzo 
dei ricordi e delle tracce (oggetti, segni nel paesaggio, ecc.), si articolano nella memoria come parte integrante di un passato che non ha perso il contatto con il presente, un passato che non si è irrigidito nelle rappresentazioni storiche. Per questo motivo Rigoni Stern afferma, citando Walter Benjamin ${ }^{8}$, di considerare se stesso come un narratore orale più che un romanziere, cioè come uno che trova le sue storie nel tessuto vivo dell'oralità, dell'esperienza, della memoria condivisa, anziché inventarle nella solitudine del proprio isolamento. Scrive Benjamin che "l'esperienza che passa di bocca in bocca è la fonte a cui hanno attinto tutti i narratori. E fra quelli che hanno messo per iscritto le loro storie, i più grandi sono proprio quelli la cui scrittura si distingue meno dalla voce degli infiniti narratori anonimi" (Benjamin, Angelus novus, 248). Nella modernità la scrittura si è imposta sull'oralità spesso portando il materiale stesso delle narrazioni anonime nel libro, permettendo ai miti, alle leggende e alle tradizioni di trasmettersi oltre la cerchia della comunità locale e di fissarsi in una forma stabile su cui le variazioni non operano più. Non perciò alla rappresentazione del passato rimane aperta solo la via del verosimile romanzseco: "Ritorno sul Don" non osserva il concetto di verosimile manzoniano, in cui al vero storico si aggiungeva il verosimile poetico come invenzione vincolata. Il verosimile rigoniano è opposto a quello manzoniano dal momento che il poeta non si pone al di sopra del passato che narra, bensì vi partecipa mediante l'esperienza della traccia, attraverso la quale quel passato rimane in comunicazione col presente mentre questo si pone in apertura alla ricezione e alla comprensione di quello.

Già prima di "Ritorno sul Don" il tema dell'affioramento del passato sotto l'apparenza della realtà presente apparve ne Il bosco degli urogalli (1962), dove la quiete del ritorno alla pace è solo apparente, come scrivono Buzzi (Invito alla lettura di Rigoni Stern, 45) e poi lo stesso Rigoni Stern nella prefazione all'edizione scolastica del 1970:

Ogni tanto, tra questi racconti così semplicemente paesani, appaiono dei ricordi improvvisi richiamati alla memoria da particolari situazioni o da cose: sono brevi episodi vissuti in guerra e che riaffiorano come a voler rammentare che non sempre vi è stata pace e serenità e come queste siano cose da difendere e conquistare giorno per giorno" (Rigoni Stern, Il bosco degli urogalli, 1970, 7)

Sono le situazioni e le cose di ogni giorno ad attivare improvvisamente

${ }^{8}$ In un'intervista del 1980, ora in Motta, Mario Rigoni Stern,11. 
la memoria e a trascinare verso la superficie i ricordi del passato di guerra, ricordi che irrompono nella pace quotidiana angosciosamente, impedendo di rifugiarsi nell'idillio, e ricordano che la serenità e il progresso sono fragili ed esposti alla distruzione e alla violenza, come una "tregua"9.

Con Ritorno sul Don il tema della traccia viene elaborato in un'architettura poetica precisa, un percorso che inizia nel cuore della campagna di Russia (i primi quattro racconti), per poi passare a narrazioni e reminiscenze di singolari episodi del tempo di guerra ("La segheria abbandonata", "Bepi, un richiamato del ' 13 " e "Un ragazzo delle nostre contrade"), fino a "La scure" (dedicato non a caso a Primo Levi), racconto sul dolore e sull'angoscia di un ritorno alla pace che in realtà è solo una "tregua", e a "Ritorno sul Don". Questo racconto sposta l'azione nel presente della cronaca mostrando la Russia ormai pacifica degli anni Settanta (rivelando anche un preciso e parziale modo di guardare la Russia di quegli anni) e porta un estremo saluto ai "compagni caduti e rimasti per sempre in quelle steppe, e anche un ringraziamento alla gente dei villaggi e delle isbe" (Rigoni Stern, Il sergente nella neve, 1990, 292), mentre la memoria crea una rete di richiami fra il presente della scrittura e il passato, che affiora per mezzo di simboli e metafore, come quella della neve, nell' incipit.

Ogni anno, quando cadeva la prima neve e dalla finestra che guarda gli orti vedevo tetti e montagne imbiancarsi, mi prendeva una malinconia che stringeva il cuore e mi isolava da tutto il resto... I primi anni prendevo gli sci e andavo. Andavo da solo dove non avrei incontrato nessuno. Nessuno, tranne quello che avevo lasciato là. (Rigoni Stern, Il sergente nella neve, 1990, 279)

Che il passato evocato non è il tempo dell'infanzia o della giovinezza, lo rivela l'ultima frase alludendo alla guerra conclusa, della quale si protrae nella memoria l'eco del mutismo e dell'isolamento del reduce che non riesce a esternare e condividere le proprie esperienze dolorose. Si crea una continuità intima fra l'evento del passato, che sembra trascorso e confinato in una storia conclusa, e il fenomeno della neve, che ogni anno torna e si ripete con la regolare necessità delle stagioni. Apparentemente potrebbe sembrare che Rigoni Stern accosti due tempi inconciliati, quello della sto-

${ }^{9}$ Nel medesimo anno Primo Levi pubblicava La tregua, la cui conclusione condensa la medesima angoscia: il doppio sogno del ritorno a casa e alla pace cui si sovrappone il sogno del Lager, in cui risuona il comando dell'alba, la "parola straniera, temuta e attesa: alzarsi, "Wstawaç" (Primo Levi, La tregua, 395), a indicare che le ferite della memoria erano rimaste occultate sotto l'aspetto sereno della pace. 
ria e quello della natura, per costituire una temporalità straniante e ripiegata nell'introspezione psicologica. Ma è appunto un'apparenza, perché la ripetizione della neve dilata il passato fino ad assorbire l'io narrante, sollevando l'enigma angoscioso: se la neve ritorna ogni anno uguale, perché i morti giacciono nel passato e nella memoria, e perché l' "io" del passato non è qui ma è rimasto laggiù?? Nello iato sorge la coscienza di una separazione componibile soltanto come richiamo, che si cela in una citazione: "Nessuno", senhal dietro cui il narratore mostra se stesso, evoca un "sé" assente e lo richiama nella sua immagine di fantasma, perché esso è rimasto là in Russia, appartiene ad un passato che non è più qui ora, ma che in ogni momento può tornare nelle immagini della memoria e sovrapporsi per somiglianza a quelle del presente, alla neve, all'inchiostro:

Certe volte che ero in ufficio a trascrivere le volture catastali sui vecchi registri mi pareva che il nero dell'inchiostro ferro-gallico sulla pagina fosse come la colonna in ritirata nella steppa. E mi capitava pure di scrivere nomi di compaesani che non erano ritornati... Così ogni volta che nevicava era come morire un poco. (Rigoni Stern, Il sergente nella neve, 1990, 279)

La memoria funziona per analogie, e così come la riga nera sulla carta è immagine allegorica della rotta di Russia, allo stesso modo la neve che scende rievoca i fantasmi dei morti e dei dispersi, muti e anonimi come quel "sé-Nessuno" lasciato in Russia dallo scrittore. La neve, simbolo dell'infinita capacità della physis di rigenerarsi e di ripetersi nei suoi cicli, nella memoria evoca l'immagine metaforica di un'altra realtà già vissuta, che sempre torna simile nella sua forma (il ricordo-incubo della colonna nella neve) e sempre però manifesta qualcosa di diverso, un senso che si rinnova ad ogni rievocazione. Questo perché pur rimanendo lo stesso (ipse), colui che ricorda e dipana la memoria nel tempo non resta il medesimo (idem): come i compagni perduti, anche il Rigoni Stern che a quel tempo fu con loro in Russia non è più. Lo scritto del 1973 insegue l'essenza vera della testimonianza, di linguaggio postumo, il cui narratore si pone rispetto a se stesso e al proprio passato come un sopravvissuto che lascia dietro una parte di sé, consapevo-le che soltanto nella testimonianza, appunto, quella porzione di essere caduta nel silenzio e nell'oblio riceverà un volto, una voce, un nome. Al di fuori del linguaggio, "io" è Nessuno, assenza silenziosa, un non-morto, e forse è questo il senso della domanda enigmatica: "fu per questo vivere, forse, che un mattino di dicembre il cuore si fermò?" (280). Un vivere che, ad ogni nuovo ritorno del ricordo, è anche un morire, logorante, ango-sciante: ha ragione Buzzi nel sostenere che non c'è 
serenità in questa opera, bensì il senso di un'oppressione che incombe.

Tuttavia non c'è dubbio che il narratore del presente e quel "Nessuno" del passato siano momenti e aspetti diversi di una medesima esistenza, di un individuo che è contemporaneamente reduce, scrittore ("ero pur sempre quel tale che aveva scritto Il sergente"; 280), impiegato del catasto di Asiago, infine pensionato che, dopo un infarto, lascia il mestiere e con la liquidazione torna in Russia. La vita appare intelligibile nella forma autobiografica che trattiene assieme il fascio dei ricordi, degli episodi e delle esperienze vissute con il filo rosso della coscienza di sé. Nella forma autobiografica, direbbe Gadamer, la vita appare "come" qualcosa. Eppure questa unità è attraversata dalla discontinuità, oscuramente. Le esperienze, le diverse identità, i ricordi appaiono ordinatamente coerenti nel momento in cui si fanno presenti alla coscienza, nel racconto, nel medium del linguaggio, eppure qualcosa divide il "qui e ora" dal "laggiù allora", e ciò che era vivo allora non lo è più adesso. "Ritorno sul Don" non è soltanto, come scrive Buzzi, "una sorta di bagno purificatore nel tempo e nello spazio della memoria, una comunione fisica con i non sopravvissuti attraverso il contatto diretto con i luoghi e le cose" (Buzzi, Invito alla lettura, 69-70), scaturito dalla necessità di espiare il senso di colpa del sopravvissuto: piuttosto si tratta di un'avventura della memoria, che risale al contrario il proprio corso per scoprire se ciò che era morto nel passato (la vita quotidiana nella Russia del 1942-43, distrutta dalla guerra) può essere invece vivo oggi. $\grave{E}$ un'avventura nella speranza più che nell'espiazione, da ciò l'assenza di serenità che permea l'inizio del racconto e l'atmosfera della raccolta in generale, perché in gioco fino alla fine sta una scommessa rischiosa: se ogni passato è la radice di un futuro, quale futuro può essere sorto dalla devastazione della guerra? Così il movimento dai singoli episodi alla vita come articolazione compiuta di un senso generale, è simbolico e allegorico: tanto, infatti, le immagini della Russia attuale fungono da simboli di una Russia altrettanto vera nella memoria, quanto il racconto nella sua interezza è allegoria della ricerca del senso che quel passato continua a produrre nel presente attraverso le sue tracce, a cominciare dal viaggio attraverso i boschi e le pianure dell'est:

L'autunno è meraviglioso di colori e questi boschi sembrano i miei... È un mondo pulito e pacifico, ma pure sento dentro qualcosa che risale, come una paura profonda e assopita, e quando giungiamo a Lvov (la Leopoli per i polacchi, la Lemberg per i tedeschi) mi ritrovo dentro la stazione di allora, quella distrutta dai bombardamenti e le donne ebree di quel lontano 1942, e non questa ricostruita, con la gente tranquilla e serena attorno ai treni. (Rigoni Stern, Il sergente nella neve, 1990, 287) 
È l'immagine (eikon) di un'altra realtà, né perduta né scomparsa, che si sovrappone di colpo all'esperienza dell' hic et nunc trasfigurandola come possibilità e imponendo alla sua apparente serenità l'autentico e terrificante senso dell'eterno ritorno: la continua manifestazione dell'essere nelle sue possibilità infınitamente aperte, per cui l'Europa che il narratore descrive è la tranquilla terra di pace, di boschi e di vita serena, ma anche e nello stesso tempo il luogo della guerra, della distruzione che la memoria continua a mostrare. Il presente e il passato si compenetrano in una visione simbolica, il cui senso può darsi solo come ricerca, non come il ritrovamento di qualcosa di già dato ma perduto: "il treno corre nella notte e non dormo. Allora eravamo in tanti dentro i vagoni dalle porte spalancate e si stava distesi tra armi e zaini. Ma ha un senso andare alla ricerca di quel tempo?" (288-289). Non c'è un tempo del qui e ora distinto da un tempo dell'allora, è il linguaggio ad articolarne la distanza e la separazione, e nel nulla che li separa ne coglie il senso (quel nulla, iato, che divide l'essere dall'apparire), del quale l'autobiografia si fa articolazione e manifestazione.

Perciò il passato, sovrapponendosi al presente, tuttavia non lo incontra mai in identità, come se una cesura invisibile distinguesse le due immagini:

Certo, questa che vedo non è la Russia dei miei ricordi: la città è pulita, ordinata, fresca; e la gente se ne va tranquilla per le belle strade alberate. Ma questo parlare mi riporta i compagni con i quali ho diviso i lunghi mesi nei Lager tedeschi, e i ragazzi che se ne vanno a scuola allegri altri ragazzi che in tristissimi tempi si erano aggregati alla cucina del Cervino per poter sopravvivere. (Rigoni Stern, Il sergente nella neve, 1990, 289)

La distanza è la necessità fondamentale affinché il passato in quanto. esperienza vissuta, la cui comprensione e articolazione è la chiave per la costituzione di un senso della vita, non appaia come una monade isolata ma come parte di un insieme generale: perciò la scrittura autobiografica si compone come mosaico, è allegoria di una totalità che non è il "sé", ma la manifestazione di un essere che si rigenera con un movimento di emersione/immersione, avvicinamento/allontanamento, scomparsa/ritorno, di cui il "sé" è soltanto una parte, un momento, una prospettiva.

Sotto l'epidermide della realtà quotidiana si svela l'essenza segreta di qualcosa di cui il presente e il passato non sono che possibilità: perciò è strano "vedere la gente tranquilla vicino alla chiesa ad aspettare la corriera, e non sentire i carri armati e le raffiche dei mitra" (305), mentre le isbe e le donne sedute sulle panchine evocano le medesime immagini che "apparvero dentro il fumo della tormenta" (306) durante la ritirata. Strano e naturale nel medesimo tempo, perché la verità non appartiene all'ap- 
parenza, ma è per mezzo di essa che si mostra, e la ricerca per cui il narratore giunge a vedere la verità dietro l'apparenza consiste nel rimanere aperto sulla realtà che gli si para innanzi e lasciare che essa manifesti ciò che in lei si cela, il senso del suo passato, del suo dolore, del suo oblio ${ }^{10}$. Ma in tale passato il narratore stesso è coinvolto e il suo dolore è solidale, è un compatire l'esperienza della finitezza mediante l'apertura ad un essere che è sempre eccedente rispetto alle sue manifestazioni. Apertura resa possibile dalla memoria che scorge il passato nel presente e prevede in ogni ricordo un annuncio del presente, in circolarità, promessa di ritorno.

Ma che cosa è questo essere, se non coincide con le rappresentazioni della memoria né con l'esperienza sensibile del presente, né tanto meno con il "sé" autobiografico? L'essere che affiora dalle cose e si manifesta mediante la sovrapposizione nella memoria di somiglianze e diversità, è la physis, la vita che l'uomo condivide con le altre creature della natura, con i boschi e le bestie, pienezza priva di principio e di fine. Alla testimonianza dell'avvento di questo essere la parola di Rigoni Stern rimane aperta, seguendo non il desiderio psicologico della redenzione dalla colpa individuale del sopravvissuto, ma indicando un orizzonte escatologico di risanamento della ferita, questa sì generale e "cosmica", della guerra, "esperienza che separa" (Polato, "La 'memoria", 391) l'uomo dalla natura e fa "violenza a tutto quanto c'è nell'universo" (Motta, Mario Rigoni Stern, 6). La riconquista della pace mediante l'esperienza mistica della natura è allegoria della redenzione dal male della violenza, e della ricomposizione dell'infranto equilibrio fra il tempo interrotto dell'esistenza umana (storica) e quello infinito della physis. Da qui deriva il tono nostalgico ed elegiaco, ma non idilliaco, dell'opera. Nella scrittura di Rigoni Stern il passato e il presente stanno reciprocamente in una relazione simbolica, in cui il presente funge da traccia di un passato che ritorna nelle proprie immagini, nei racconti, nella memoria. Allora il presente stesso è sempre il passato di un futuro in cui tornerà senza sosta, e le sue rappresentazioni lo tramanderanno: nel circolo autobiografico la rappresentazione del passato svela la potenza della vita che non si esaurisce mai e che nella morte conosce soltanto un passaggio necessario. Di conseguenza, questo circolo non si chiude sul soggetto, ma conosce in esso il momento dell'interruzione, del disvelamento. Rigoni Stern infatti, ritrovando le tracce del suo stesso passaggio, non trova più soltanto se stesso, ma coloro che sono caduti nel

\footnotetext{
${ }^{10}$ Tale accezione l'essenza della verità e dell'opera d'arte è quella di Martin Heidegger di cui si richiamano Dell'essenza della verità (1943) e "Lorigine dell'opera d'arte" (1936).
} 
silenzio:

Una nuvola di corvi si alza da un arato senza confini. Ma laggiù, tra pochi alberi del colore dell'autunno, in un grande silenzio, due villaggi sembrano confondersi e impastarsi con l'aria e la terra: Nikitova e Arnautovo. Non mi posso sbagliare. No, non mi sono sbagliato. Cammino fuori dalla pista. Capitano Grandi del Tirano, dormi in questa pace. Ti porto i saluti dei superstiti del tuo battaglione, di Nuto Revelli e di tutti gli alpini della Tridentina. Dormite in pace amici valtellinesi, in questo silenzio, in questa terra nera, in questo autunno dolcissimo. Chino la testa e poi faccio un cenno con la mano: - Ci ritroveremo un giomo. Arrivederci. (Rigoni Stern, Il sergente nella neve, 1990, 299)

Emblematico è il movimento della scena, dal paesaggio all'io, al tu, al voi, fino al commosso noi della promessa di ritrovarsi uniti nella mortenarrazione. La memoria autobiografica rivela che la medesima realtà è una possibilità sempre in movimento e diversa, e mentre alla sua superficie si svela l'essere (la physis) sotto l'apparenza della vita quotidiana, sotto la superficie delle immagini l'essere si nasconde e con esso il senso di quella vita che si dipana, scorre, svanisce"1: altri volti ha mostrato la physis, presenti nella memoria, in cui quella campagna pacifica è il luogo della guerra, della morte e del dolore. La parola dell'io diventa in tal modo la testimonianza di un passato condiviso con altri che non sono più e di cui il testimone è responsabile, nel presente con altri superstiti (Nuto Revelli) che vivono e testimoniano a loro volta quel passato, in forme sempre diverse.

L'arrivo a Nikolajevka coincide con il ritrovamento di ciò che, andando alla ricerca del senso del passato, irriducibilmente sfugge e non si lascia afferrare e comprendere, la ragione della morte dei compagni e degli amici in una battaglia, in una guerra, che, di fronte al mistero della vita che eternamente rinasce appare insensata, assurda e inutile. "Ma come abbiamo potuto?", "ma è possibile? Ma come è stato possibile?" (301), si domanda il narratore, che trasforma l'esperienza vissuta in una testimonianza di valo-

${ }^{11}$ Che l'essere non si dia nell'apparenza degli enti, ma che da questa sia reso intuibile nella sua profondità, è il nocciolo dell'ontologia antimetafisica di Martin Heidegger, e della sua celebre metafora della "radura", cioè quel luogo in cui il fitto dirada, mostrando solo così e in un istante l'essenza della "fittezza". La nozione di "disvelamento dell'essere", anticipata già in Essere e tempo (1927), è poi al centro di importanti scritti heideggeriani come i già citati Dell'essenza della verità e L'origine dell'opera d'arte, e alla radice dell'intera produzione del dopoguerra. 
re generale e compie, mediante la rappresentazione del proprio passato, anche il gesto etico di rendere giustizia ai morti e ad una vita che è stata oltraggiata dalla violenza, ma non lo fa con la pretesa di pronunciare una parola assoluta e totale, bensì soltanto momentanea, anch'essa segno di un passaggio che sarà a sua volta superato:

Ecco, da qui, a ogni gruppo di isbe è legata la nostra storia... Siamo passati per ogni pista e i nostri nomi gridati nella tormenta di queste steppe. Morte, speranza, disperazione, fatalismo. Chi potrebbe dire tutto? Nessuno. Nessuno saprà tutto. E per tutti e per ognuno una storia diversa. Ed eravamo tanti. (Rigoni Stern, Il sergente nella neve, 1990, 304)

L'autobiografismo ha valore di testimonianza e la sua portata di verità è generale, ma soltanto nella misura in cui esemplifica una possibilità di essere che verosimilmente può dire ciò che i morti non testimonieranno mai più. Nessuno sa tutto né lo saprà mai, perché la totalità non appartiene all'umano ma lo trascende, è prerogativa di una vita che anela ad essere sempre più di quel che è tornando eternamente su se stessa. Ma Nessuno è anche l'inseparabile di ognuno. Ecco il paradosso: nel tempo finito della vita di ognuno, l'essere infinito non si lascia irretire, rimane indicibile, incontenibile. Ma niente di ciò che vive è fuori della physis, perciò tutto eternamente si ripete. Non come soggetto, finito, limitato, il narratore può dire tutto, ma come Nessuno egli sperimenta la morte su se stesso, e ancora vivo sa cosa significa trascendere la finitezza ${ }^{12}$.

Qual è la condizione affinché la vita sia totalità? Come può la vita eternamente tornare a se stessa senza interrompersi? La morte è l'evento cardine, il ritorno per eccellenza della vita al tutto, è la morte che borda l'autobiografia e ne detta il limite estremo oltre cui il narratore non può spingersi, essa è "la sanzione di tutto ciò che il narratore può raccontare. Dalla morte egli attinge la sua autorità" (Benjamin, Angelus novus, 259). Il ritorno alla baita è per Rigoni Stern il simbolo della morte in cui ci si ricon-

${ }^{12}$ Ciò che qui è assicurato al narratore, Platone lo assicura al filosofo, soprattutto nel Simposio, ovvero a colui che sa staccarsi dalle cose terrene e assecondare la mania erotica, ovvero lo slancio a conquistare ciò di cui soffre la mancanza nel proprio essere: l'immortalità e la perfezione, attributi divini che ogni uomo condivide con gli dei per mezzo dell'anima. Escludendo questo orizzonte metafisico, e puntando più schiettamente sulla forza della rappresentazione come "volontà di potenza" e "disvelamento dell'essere", riteniamo che l'arte di Rigoni Stern si liberi del soggettivismo da un lato e dell'idealismo dall'altro, in favore di una concezione del cosmo come organismo vivente e giustificato dal suo stesso principio vitalistico. 
giunge al tutto, e il nostos dalla Russia a casa diventa "una allegoria della maturità, in cui il vagabondare, l'esperienza, hanno un termine che richiama: la casa, dove raccontare, la morte dove farsi racconto" (Motta, Mario Rigoni Stern, 7), principio della narrazione e della memoria perché, come dice ancora Benjamin, la vita vissuta "assume forma tramandabile solo nel morente" (Benjamin, Angelus novus, 258). Una volta tornato a casa, il reduce può farsi narratore di sé stesso solo in quanto morente: esso ha lasciato laggiù, nel luogo dell'esperienza che si appresta a narrare, ciò che è stato e che in attesa di essere detto, narrato, trasceso in una forma, è Nessuno13. Il ritorno alla baita è l'attraversamento di una soglia oltre la quale il narratore è diviso da se stesso, è a distanza da sé e può allora cogliersi come un altro, giudicarsi, rappresentarsi.

"Ritorno sul Don" è lo spartiacque fra una prima fase di memorialistica caratterizzata dalla narrazione diretta del passato con l'affondamento del punto di vista narrativo interamente dentro la materia dei ricordi, e una seconda fase in cui si afferma in maniera più evidente una concezione del passato come un momento possibile dell'essere, il cui senso sempre sfugge e che si manifesta soltanto quando l'istante della morte lo congela in una forma. Non c'è frattura fra "Ritorno sul Don" e le opere precedenti, ma un approfondimento sempre più consapevole della ricchezza inesauribile delle risorse estetiche della memoria: già ne Il sergente nella neve, Il bosco degli urogalli e Quota Albania la narrazione era tesa verso la ricomposizione di un equilibrio armonico e compenetrato, pacificatore, fra la coscienza umana della propria finitezza e la vita che si dissolve e sempre torna a rinascere. Tre volte Rigoni Stern è tornato dalla Russia, ne ha scritto a più riprese, mostrando una realtà sempre nuova eppure sempre uguale, accrescendo l'essere di quella vita rappresa in esperienza e nella forma dei racconti, eppure sempre più grande e ricca di quanto le forme potessero essere:

Ecco, sono ritornato a casa ancora una volta; ma ora so che laggiù, quello tra il Donetz e il Don, è diventato il posto più tranquillo del mondo. C'è una grande pace, un grande silenzio, un'infinita dolcezza. La finestra della mia stanza inquadra boschi e montagne, ma lontano, oltre le Alpi,

${ }^{13}$ Senza dilungarci eccessivamente, si pensi soltanto all'importanza che un tema come il nostos ha nella letteratura italiana del dopoguerra, e spesso con espliciti riferimenti alla figura di Ulisse. Si va dai poemetti di Giovanni Pascoli "Il sonno di Odisseo" (1899) e "L'ultimo viaggio" (1899), a Capitano Ulisse di Alberto Savinio (1925), fino a La luna e $i$ falò di Pavese (1950) passando per Conversazione in Sicilia di Vittorini (1941). In epoca più recente si ricordi anche Horcynus Orca di Stefano D'Arrigo (1975). 
le pianure, i grandi fumi, vedo sempre quei villaggi e quelle pianure dove dormono nella loro pace i nostri compagni che non sono tornati a baita. (Rigoni Stern, Il sergente nella neve, 1990, 317)

Un senso è stato trovato, una redenzione è stata realizzata, e quei luoghi di guerra e morte sono ora riconsegnati alla memoria come luoghi di pace e dolcezza. E nella chiusura che è un piccolo inno all'immaginazione, la distanza è coperta oltre gli spazi immensi dell'Europa dalla vista che ritrova sempre i luoghi dell'esperienza, ma trasfigurati, ormai quieti e silenziosi, dove riposano i morti che non sono tornati a casa: coloro che non hanno attraversato la soglia metaforica per fare di se stessi storie viventi, ma sono consegnati al silenzio, tracce nascoste in forma di ricordi di un passato violento. Di essi è custode il testimone con la sua memoria, "la facoltà epica per eccellenza" (Benjamin, Angelus novus, 261), la fonte della voce che parla per tutti perché parla di Nessuno: "solo mercè una vasta memoria l'epica può, da un lato, appropriarsi il corso delle cose, e, dall'altro, riconciliarsi col loro scomparire, con la potenza della morte" (301302). E la memoria a cui Rigoni Stern attinge la forza del proprio narrare è potente poiché in essa non si conclude e risolve un circolo soggettivo, ma confluisce la voce remota di altri narratori che, dall'Altipiano alla steppa, narrano gli infiniti volti della stessa vita, che sempre torna a se stessa, oltre gli individui, oltre la storia.

National University of IRELAND, Cork

\section{OPERE CITATE}

Affinati, Eraldo. "Mario Rigoni Stern: la responsabilità del sottufficiale”, pp. xi-li in Rigoni Stern, Mario, Storie dall'altipiano, Milano: Mondadori, 2003.

Aristotele. Poetica. 8a ed. Milano: Rizzoli, 1996.

Benjamin, Walter. Angelus Novus. Torino: Einaudi, 1995.

Buzzi, Michele. Invito alla lettura di Rigoni Stern. Milano: Mursia, 1985.

D’Arrigo, Stefano. Horcynus Orca. Milano: Mondadori, 1975.

Eliade, Mircea. Il mito dell'eterno ritorno. Torino: Borla, 1968.

Gadamer, Hans-Georg. Verità e metodo. 14a ed. Milano: Bompiani, 2004.

Heidegger, Martin. Dell'essenza della verità. Milano: Bocca, 1952.

- "L'origine dell'opera d'arte". Sentieri interrotti. Firenze: La Nuova Italia, 1968. 3-69.

Levi, Primo. Opere. 2 voll. Torino: Einaudi, 1997.

Mauron, Charles. Dalle metafore ossessive al mito personale. Introduzione alla psicocritica. Milano: Il Saggiatore, 1966.

Motta, Antonio. Mario Rigoni Stern. Firenze: La Nuova Italia, 1982. 
Nietzsche, Friedrich. Cosi parlò Zarathustra. Un libro per tutti e per nessuno. Milano: Adelphi, 1976.

- Crepuscolo degli idoli, ovvero come si filosofa col martello. 8a ed. Milano: Adelphi, 2002.

_. La gaia scienza. 2a ed. Milano: Adelphi, 1991.

La volontà di potenza. Frammenti postumi. Milano: Bompiani, 1992.

Pascoli, Giovanni. Poemi conviviali. Milano: Mondadori, 2002.

Pavese, Cesare. La luna e i falò. Torino: Einaudi, 1950.

Platone. Fedro. Milano: Mondadori, 2005.

Simposio, Apologia di Socrate, Critone, Fedone. Mondadori: Milano, 2004

Sofista. Milano: Rizzoli, 2007.

Polato, Lorenzo. "La 'memoria' di Rigoni Stern". Studi Novecenteschi 27, 60 (dicembre 2000): 385-398.

Ricoeur, Paul. Ricordare, dimenticare, perdonare: l'enigma del passato. Bologna: II Mulino, 2004.

Rigoni Stern, Mario. Il bosco degli urogalli. $3^{\mathrm{a}}$ ed. Torino: Einaudi, 1967.

- Il bosco degli urogalli. Torino: Einaudi, 1970.

. "La bottiglia ritrovata". Aspettando l'alba e altri racconti. Torino: Einaudi, 2005. 32-43.

_. "Cartoline". Sentieri sotto la neve. Torino: Einaudi, 1999. 78-82.

—. Quota Albania. 2a ed. Torino: Einaudi, 2003.

_. "Ritorno sul Don". Il sergente nella neve - Ritorno sul Don. Torino: Einaudi, 1990. 277-317.

- Il sergente nella neve. Torino: Einaudi, 1953.

- Le stagioni di Giacomo. Torino: Einaudi, 2005.

—. Storia di Tönle - L'anno della vittoria. $10^{\mathrm{a}} \mathrm{ed}$. Torino: Einaudi, 2003.

"Tre patate lesse". Il sergente nella neve - Ritorno sul Don. Torino: Einaudi, 1990. 211-221.

Lultima partita a carte. Torino: Einaudi, 2002.

Savinio, Alberto. Capitano Ulisse. Milano: Adelphi, 1989.

Vittorini, Elio. Conversazione in Sicilia. Milano: Bompiani, 1941. 